\title{
Cognitive load theory as a framework for simulation-based, ultrasound-guided internal jugular catheterization training: Once is not enough, but we must measure it first
}

To the editor: We wish to thank Drs. de la Fuente and Altermatt for their careful review of our paper, and for their thoughtful comments. They highlight some important limitations of our study and areas for future research.

We believe the difficulty novices experience while learning ultrasound-guided procedures reflects the demands on working memory of interpreting a two-dimensional ultrasound image while simultaneously manipulating an ultrasound probe with one hand, and a syringe and needle with the other. Our curriculum addressed this challenge by segmenting these skills into smaller part tasks to respect the working memory capacity of the learners.
The part tasks chosen were based on our experience, but we agree that more work is needed to determine the optimum strategy for task segmentation by measuring the cognitive load associated with different part task options.

Drs. de la Fuente and Altermatt also correctly point out that the gains in performance we observed could have been a result of distributed deliberate practice, and not necessarily a result of our progressive part practice strategy. This was acknowledged in our paper under limitations, and in fact we agree that distinguished deliberate practice was likely the most important determinant of proficiency. Cognitive load theory was used to describe the rationale for our curriculum because it provides insight into why this high-volume, deliberate practice is necessary, and why we should not expect proficiency in this skill after one session.

Again, we thank Drs. de la Fuente and Altermatt for their comments, and for furthering the discussion around skills training in the simulation lab.

On behalf of the study authors,

\section{Robert McGraw,}

Queen's University, Department of Emergency Medicine

Keywords: Simulation, cognitive load theory 